\title{
TIGHTER BOUND FUNCTIONS FOR NONCONVEX FUNCTIONS OVER SIMPLEXES
}

\author{
OuAnes Mohand*
}

\begin{abstract}
In this paper, we propose new lower and upper bound functions which can be used in computing a range of nonconvex functions over simplexes of $R^{n}$, or for solving global optimization problems over simplexes. We show that the new bounding functions are tighter than the classical bounding functions developed in the $\alpha \mathrm{BB}$ method and the $\mathrm{QBB}$ method.
\end{abstract}

Mathematics Subject Classification. 65K05, 90C30, 90C34.

Received March 23, 2020. Accepted August 6, 2020.

\section{INTRODUCTION}

We consider the following problem

$$
(P)\left\{\begin{array}{c}
\min f(x) \\
x \in \Delta \subset R^{n}
\end{array}\right.
$$

where $f$ is a $C^{2}$-nonconvex function and $\Delta$ is an $n$-simplex of $R^{n}, \Delta=\left\{s^{0}, s^{1}, \ldots, s^{n}\right\}$ is the convex hull of $(n+1)$ points $s^{i} \in R^{n}$ that do not lie in a hyperplane of dimension less than $n$. (e.g., a 2 -simplex is a triangle, while a 3 -simplex is a tetrahedron).

Several methods have been studied in the literature for global optimization problems over simplices. These methods are useful for example if, (i) the feasible set is a simplex, (ii) the feasible set is defined by linear constraints, (iii) the feasible set is a box which can be divided in simplexes. Let us mention some works, in [15] a QBB method is presented which consists of constructing a convex quadratic underestimator over simplices and used in branch and bound algorithm for solving global optimization problems. In [6], a range bound of functions over simplexes is presented, and the interval analysis method [15] for the gradient of the objective function is used to construct the bounds. In [10], the DISIMPL (DIviding SIMPLices)) is used for Lipschitz global optimization which is an extented algorithm of DIRECT (DIviding RECTangles) algorithm. For the function evaluations, it consists of two methods, the first method uses the vertices of the simplex, and the second method uses the centroid of the simplex. If the feasible set is an hyperrectangle and the objective function is symmetric then the feasible set is reduced to a simplex by adding some linear constraints (Avoidance of symmetries of objective function). In [11], the DISIMPL algorithm with the simplicial partitionning is adapted for solving Lipschitz global optimization problems with linear constraints. The method developed in [12] consists of reducing the

Keywords. Global optimization, range of functions, convex lower bound function, concave upper bound function.

LAROMAD, Département de Mathématiques, Faculté des Sciences, Université Mouloud Mammeri, Tizi-Ouzou, Algérie.

*Corresponding author: ouanes_mohand@yahoo.fr 
initial hyperrectangle to the unit hypercube and subdivide the resulting hypercube into $n$ ! simplices by using the face to face vertex triangulation. If the objective function is evaluated at the vertices simplex then the bissection method is used, if it is evaluated at the centoid of the simplex then the trisection method is used. In [4], the so-called monotonicity test used in interval branch and bound algorithm over a box is applied to simplicial branch and bound algorithm over a simplex. In [8], a refinement method which reduces the magnitude of $\alpha$ values given by the scaled Gerschgorin method is presented and thus create a tighter convex underestimators for the $\alpha \mathrm{BB}$ algorithm. In [7], a transformation of the original function into a $\mu$-subenergy function is used and the $\alpha \mathrm{BB}$ underestimators for the new function are applied. They propose a methodology to build $\alpha \mathrm{BB}$ underestimators which may be arbitrarily tight (i.e. the maximum separation distance between the original function and its underestimator is arbitrarily close to 0) in some domains that do not include the global solution. In [3], a branch and bound algorithm is presented for Hölder functions. In [14], the improved linear bounding function is used and discard regions which do not contain the global minimum by the pruning step(outer) as in [13]. In [2], a review of recent advances in global optimization is presented.

The efficiency of a method for solving global optimization problems is in the construction of tight underestimator and to discard big regions which do not contain the global minimum as quickly as possible in branch and bound algorithm.

The contribution of our paper is to propose a new lower function which is tighter than the quadratic lower bound function developed in [15] and the lower bound function developed in the classical $\alpha \mathrm{BB}$ method which was already proposed in [1]. In the same way, we propose a tighter upper bound function. Our contribution is an extension of the work developed in [9].

The structure of the paper is as follows. The two lower bound functions developed in [15] and in [1] with their properties are presented in Section 2, those functions apply other than over simplexes. In Section 3, the new lower and upper bound functions are stated with their properties. Computational results are reported in Section 4.

\section{CONVEX LOWER BOUND FUNCTIONS OVER SIMPLEXES}

\subsection{Quadratic convex lower bound function [15]}

The quadratic lower function developed in [15] on an $n$-simplex $\Delta$ is defined as follows:

$$
\operatorname{LB}_{q}(x)=\frac{K_{q}}{2} \sum_{i=1}^{n} x_{i}^{2}+\sum_{i=1}^{n} \alpha_{i} x_{i}+\alpha_{0}
$$

where $K_{q} \geq \max \left\{0, \lambda_{\max } H f(x)\right\}$ and $\lambda_{\max } H f(x)$ is the maximal eigenvalue of the Hessian matrix of $f(x)$.

The properties of this lower bound function are:

(1) $\operatorname{LB}_{q}(x)$ coincides with the function $f(x)$ at the vertices of the simplex $\Delta$.

(2) $\operatorname{LB}_{q}(x)$ is convex.

(3) $\operatorname{LB}_{q}(x)$ is a lower bound function of $f(x)$ on $\Delta$ which implies that

$$
\operatorname{LB}_{q}(x) \leq f(x), \forall x \in \Delta .
$$

For more details see [15].

We propose to write this quadratic lower bound function as a difference of a linear function and a quadratic concave function as follows:

$$
\operatorname{LB}_{q}(x)=L_{h} f(x)-\frac{K_{q}}{2} Q_{h}(x),
$$

where

$$
L_{h} f(x)=\sum_{i=1}^{n} \beta_{i} x_{i}+\beta_{0},
$$


is a linear interpolant of $f(x)$ on the simplex $\Delta$ which coincides with $f(x)$ at the vertices of $\Delta$, and

$$
Q_{h}(x)=-\sum_{i=1}^{n} x_{i}^{2}+\sum_{i=1}^{n} \gamma_{i} x_{i}+\gamma_{0}
$$

is a quadratic concave function which vanishes at the vertices of $\Delta$.

We will denote by $V(\Delta)$ the set of vertices of $\Delta$.

The parameters $\beta_{i} ; i=0, . ., n$ are obtained by solving the following linear system:

$$
L_{h} f\left(s^{j}\right)=\sum_{i=1}^{n} \beta_{i} x_{i}^{j}+\beta_{0}=f\left(s^{j}\right), j=0,1, . ., n, s^{j}=\left(x_{1}^{j}, . ., x_{n}^{j}\right) \in V(\Delta) .
$$

and the parameters $\gamma_{i} ; i=0, \ldots, n$ are obtained by solving the following linear system:

$$
Q_{h}\left(s^{j}\right)=-\sum_{i=1}^{n}\left(x_{i}^{j}\right)^{2}+\sum_{i=1}^{n} \gamma_{i} x_{i}^{j}+\gamma_{0}=0, j=0,1, . ., n, s^{j}=\left(x_{1}^{j}, . ., x_{n}^{j}\right) \in V(\Delta) .
$$

\subsection{Convex lower bound function}

We present another lower bound function on $\Delta$ as follows:

$$
\mathrm{LB}_{\alpha}(x)=f(x)-\frac{K_{\alpha}}{2} Q_{h}(x)
$$

where $K_{\alpha} \geq \max \left\{0,-\lambda_{\min } H f(x)\right\}$ and $\lambda_{\min } H f(x)$ is the smallest eigenvalue of the Hessian matrix of $f(x)$.

In the case of a right simplex, $\operatorname{LB}_{\alpha}(x)$ is exactly the underestimator of the $\alpha \mathrm{BB}$ method [1].

The properties of this lower bound function are:

(1) $\operatorname{LB}_{\alpha}(x)$ coincides with $f(x)$ at the vertices of $\Delta$ (i.e. by construction of $\operatorname{LB}_{\alpha}(x)$ ).

(2) $\operatorname{LB}_{\alpha}(x)$ is convex.

Indeed, the Hessian matrix of $\operatorname{LB}_{\alpha}(x)$ is

$$
\operatorname{HLB}_{\alpha}(x)=H f(x)+K_{\alpha} I,
$$

which is positive semidefinite because $K_{\alpha} \geq-\lambda_{\min } H f(x)$ where $I$ is the identity matrix, so $\operatorname{LB}_{\alpha}(x)$ is convex.

(3) It is a lower bound function of $f$.

Indeed, the Hessian matrix of $\left(\operatorname{LB}_{\alpha}(x)-f(x)\right)$ is

$$
H\left(\mathrm{LB}_{\alpha}(x)-f(x)\right)=K_{\alpha} I
$$

which is positive semidefinite because $K_{\alpha} \geq 0$, so $\left(\operatorname{LB}_{\alpha}(x)-f(x)\right)$ is convex on $\Delta$, moreover it vanishes at the vertices of $\Delta$ which implies that

$$
\operatorname{LB}_{\alpha}(x)-f(x) \leq 0, \forall x \in \Delta,
$$

hence $\operatorname{LB}_{\alpha}(x)$ is a lower bound function of $f(x)$ on $\Delta$.

Remark 2.1. $K_{q}$ and $K_{\alpha}$ can be computed by the method developed in [5] using the smallest hyperrectangle containing the simplex $\Delta$. 


\subsection{New convex lower bound function}

Now, we present our new convex lower bound function on the simplex $\Delta$ which is given by

$$
\operatorname{LB}(x)=\frac{K_{q} f(x)+K_{\alpha} \mathrm{LB}_{q}(x)}{K_{\alpha}+K_{q}}=\frac{K_{\alpha} L_{h} f(x)+K_{q} \mathrm{LB}_{\alpha}(x)}{K_{\alpha}+K_{q}} .
$$

\section{Proposition 2.2.}

(i) $\mathrm{LB}(x)$ coincides with $f(x)$ at the vertices of $\Delta$.

(ii) $\operatorname{LB}(x)$ is a convex function on $\Delta$.

Proof.

(i) It's obvious (i.e. by construction of $\operatorname{LB}(x)$ ).

(ii) One has

$$
\mathrm{LB}(x)=\frac{K_{\alpha} L_{h} f(x)+K_{q} \mathrm{LB}_{\alpha}(x)}{K_{\alpha}+K_{q}}
$$

which is the sum of a linear function $\frac{K_{\alpha}}{K_{\alpha}+K_{q}} L_{h} f(x)$ and a convex function $\frac{K_{q}}{K_{\alpha}+K_{q}} \operatorname{LB}_{\alpha}(x)$, so it is a convex function.

Theorem 2.3. $\operatorname{LB}(x) \leq f(x), \forall x \in \Delta$.

Proof. By using (2), one has $\forall x \in \Delta$

$$
\mathrm{LB}(x)-f(x)=\frac{K_{q} f(x)+K_{\alpha} \mathrm{LB}_{q}(x)}{K_{\alpha}+K_{q}}-f(x)=\frac{K_{\alpha}}{K_{q}+K_{\alpha}}\left(\operatorname{LB}_{q}(x)-f(x)\right) \leq 0,
$$

so

$$
\mathrm{LB}(x) \leq f(x), \forall x \in \Delta,
$$

which means that $\operatorname{LB}(x)$ is a lower bound function of $f(x)$ on $\Delta$.

In the following two theorems we will show that $\mathrm{LB}(x)$ is tighter than $\operatorname{LB}_{q}(x)$ and $\operatorname{LB}_{\alpha}(x)$ on $\Delta$.

Theorem 2.4. $\mathrm{LB}(x)$ is tighter than $\mathrm{LB}_{q}(x)$ on $\Delta$.

Proof. By using (2), one has $\forall x \in \Delta$

$$
\mathrm{LB}(x)-\mathrm{LB}_{q}(x)=\frac{K_{q} f(x)+K_{\alpha} \mathrm{LB}_{q}(x)}{K_{\alpha}+K_{q}}-\mathrm{LB}_{q}(x)=\frac{K_{q}\left(f(x)-\mathrm{LB}_{q}(x)\right.}{K_{q}+K_{\alpha}} \geq 0,
$$

so

$$
\mathrm{LB}(x) \geq \operatorname{LB}_{q}(x), \forall x \in \Delta,
$$

which implies that $\operatorname{LB}(x)$ is tighter than $\operatorname{LB}_{q}(x)$.

Theorem 2.5. $\mathrm{LB}(x)$ is tighter than $\mathrm{LB}_{\alpha}(x)$ on $\Delta$.

Proof. One has

$$
\mathrm{LB}(x)-\mathrm{LB}_{\alpha}(x)=\frac{K_{\alpha} L_{h} f(x)+K_{q} \mathrm{LB}_{\alpha}(x)}{K_{q}+K_{\alpha}}-\mathrm{LB}_{\alpha}(x)=K_{\alpha} \frac{\left(L_{h} f(x)-\mathrm{LB}_{\alpha}(x)\right)}{K_{\alpha}+K_{q}},
$$


which is the difference of a linear function and a convex function, then it is a concave function, moreover it vanishes at the vertices of $\Delta$, so

$$
\mathrm{LB}(x)-\mathrm{LB}_{\alpha}(x) \geq 0
$$

which implies that

$$
\mathrm{LB}(x) \geq \mathrm{LB}_{\alpha}(x), \forall x \in \Delta .
$$

Hence $\operatorname{LB}(x)$ is tighter than $\operatorname{LB}_{\alpha}(x)$ on $\Delta$.

Remark 2.6. This new tighter lower bound function can be used in a branch and bound algorithm for solving problem $(P)$.

In the same way as above, we will present in the next section the different upper bound functions.

\section{CONCAVE UPPER BOUND FUnCTIONS}

\subsection{Quadratic concave upper bound function}

We present the following quadratic concave upper bound function:

$$
\mathrm{UB}_{q}(x)=L_{h} f(x)+\frac{K_{\alpha}}{2} Q_{h}(x) .
$$

Theorem 3.1. $\operatorname{UB}_{q}(x)$ is a quadratic concave upper bound function of $f$ on $\Delta$.

Proof.

(i) $\mathrm{UB}_{q}(x)$ coincides with $f(x)$ at the vertices of $\Delta$ (i.e. by construction of $\mathrm{UB}_{q}(x)$ ).

(ii) By computing its Hessian, we find that

$$
\operatorname{HUB}_{q}(x)=-K_{\alpha} I .
$$

where $I$ is the identity matrix and $K_{\alpha}$ is a real nonnegative number, so $\operatorname{HUB}_{q}(x)$ is negative semi-definite which implies that $\mathrm{UB}_{q}(x)$ is a quadratic concave function.

(iii) The Hessian matrix of $\left(\mathrm{UB}_{q}(x)-f(x)\right)$ is equal to $\left(-K_{\alpha} I-H f(x)\right)$ which is negative semi-definite because

$$
K_{\alpha} \geq-\lambda_{\min } H f(x),
$$

so $\left(\mathrm{UB}_{q}(x)-f(x)\right)$ is a concave function, it also vanishes at the vertices of $\Delta$ which implies that

$$
\mathrm{UB}_{q}(x) \geq f(x), \forall x \in \Delta .
$$

\subsection{Concave upper bound function}

We present the following concave upper bound function:

$$
\mathrm{UB}_{\alpha}(x)=f(x)+\frac{K_{q}}{2} Q_{h}(x) .
$$

Theorem 3.2. $\mathrm{UB}_{\alpha}(x)$ is a concave upper bound function of $f$ on $\Delta$.

Proof.

(i) $\mathrm{UB}_{\alpha}(x)$ coincides with $f(x)$ at the vertices of $\Delta$ (i.e. by construction of $\mathrm{UB}_{\alpha}(x)$ ). 
(ii) The Hessian matrix of $\mathrm{UB}_{\alpha}(x)$ is

$$
\operatorname{HUB}_{\alpha}(x)=H f(x)-K_{q} I,
$$

where $I$ is the identity matrix, it is negative semi-definite because $K_{q} \geq \lambda_{\max } H f(x)$, so $\mathrm{UB}_{\alpha}(x)$ is a concave function.

(iii) The Hessian matrix of $\left(\mathrm{UB}_{\alpha}(x)-f(x)\right)$ is equal to $-K_{q} I$ which is negative semi-definite because $K_{q} \geq 0$, then $\left(\mathrm{UB}_{\alpha}(x)-f(x)\right)$ is a concave function which vanishes at the vertices of $\Delta$, so

$$
\mathrm{UB}_{\alpha}(x) \geq f(x), \forall x \in \Delta .
$$

\subsection{New concave upper bound function}

Now, we present our new concave upper bound function which is given by

$$
\mathrm{UB}(x)=\frac{K_{\alpha} f(x)+K_{q} \mathrm{UB}_{q}(x)}{K_{q}+K_{\alpha}}=\frac{K_{q} L_{h} f(x)+K_{\alpha} \mathrm{UB}_{\alpha}(x)}{K_{q}+K_{\alpha}} .
$$

Theorem 3.3. $\mathrm{UB}(x)$ is a concave upper bound function of $f$ on $\Delta$.

\section{Proof.}

(i) $\mathrm{UB}(x)$ coincides with $f(x)$ at the vertices of $\Delta$ (i.e. by construction of $\operatorname{UB}(x)$ ).

(ii) $\mathrm{UB}(x)$ is a concave function because

$$
\mathrm{UB}(x)=\frac{K_{q} L_{h} f(x)+K_{\alpha} \mathrm{UB}_{\alpha}(x)}{K_{q}+K_{\alpha}},
$$

is the sum of a linear function and a concave function.

(iii) $\mathrm{UB}(x)$ is an upper bound function of $f(x)$ on $\Delta$ because by using (24) and $\forall x \in \Delta$, we have

$$
\mathrm{UB}(x)-f(x)=\frac{K_{\alpha} f(x)+K_{q} \mathrm{UB}_{q}(x)}{K_{q}+K_{\alpha}}-f(x)=\frac{K_{q}\left(\mathrm{UB}_{q}(x)-f(x)\right)}{K_{q}+K_{\alpha}} \geq 0,
$$

wich implies that

$$
\mathrm{UB}(x) \geq f(x), \forall x \in \Delta
$$

In the following two theorems, we will show that $\mathrm{UB}(x)$ is tighter than $\mathrm{UB}_{q}(x)$ and $\operatorname{UB}_{\alpha}(x)$.

Theorem 3.4. $\mathrm{UB}(x)$ is tighter than $\mathrm{UB}_{q}(x)$ on $\Delta$.

Proof. By using (24), one has $\forall x \in \Delta$,

$$
\mathrm{UB}(x)-\mathrm{UB}_{q}(x)=\frac{K_{\alpha} f(x)+K_{q} \mathrm{UB}_{q}(x)}{K_{q}+K_{\alpha}}-\mathrm{UB}_{q}(x)=\frac{K_{\alpha}\left(f(x)-\mathrm{UB}_{q}(x)\right)}{K_{q}+K_{\alpha}} \leq 0,
$$

wich implies that

$$
\mathrm{UB}(x) \leq \mathrm{UB}_{q}(x), \forall x \in \Delta .
$$

So $\operatorname{UB}(x)$ is tighter than $\mathrm{UB}_{q}(x)$ on $\Delta$.

Theorem 3.5. $\mathrm{UB}(x)$ is tighter than $\mathrm{UB}_{\alpha}(x)$ on $\Delta$. 
Proof. One has

$$
\mathrm{UB}(x)-\mathrm{UB}_{\alpha}(x)=\frac{K_{q} L_{h} f(x)+K_{\alpha} \mathrm{UB}_{\alpha}(x)}{K_{q}+K_{\alpha}}-\mathrm{UB}_{\alpha}(x)=\frac{K_{q}\left(L_{h} f(x)-\mathrm{UB}_{\alpha}(x)\right)}{K_{q}+K_{\alpha}},
$$

then $\left(\mathrm{UB}(x)-\mathrm{UB}_{\alpha}(x)\right)$ is a convex function because it is the difference of a linear function and a concave function, which vanishes at the vertices of $\Delta$, so

$$
\mathrm{UB}(x) \leq \mathrm{UB}_{\alpha}(x), \forall x \in \Delta .
$$

Hence $\mathrm{UB}(x)$ is tighter than $\mathrm{UB}_{\alpha}(x)$ on $\Delta$.

Remark 3.6. By using the new lower and upper bound functions, we can compute a tighter range of nonconvex functions over simplexes.

\section{Computational Results}

We begin with the following illustrative example:

Example 4.1 ([6]). $f\left(x_{1}, x_{2}\right)=x_{1}^{2}+x_{2}^{3}, T^{0}$ has the vertices $(-1,0) ;\left(\frac{1}{2},-1\right)$ and $\left(\frac{1}{2}, 1\right)$.

We compute $L_{h} f(x), Q_{h}(x), K_{q}$ and $K_{\alpha}$, we find $L_{h} f(x)=-\frac{1}{2} x_{1}+x_{2}+\frac{1}{2}$, which coincides with $f(x)$ at the vertices of $T^{0} \cdot Q_{h}(x)=-x_{1}^{2}-x_{2}^{2}+\frac{1}{6} x_{1}+\frac{7}{6}$, which is a quaradic concave function and vanishes at the vertices of $\Delta$.

$$
K_{q}=K_{\alpha}=6 .
$$

We have

$$
\mathrm{LB}_{q}(x)=L_{h} f(x)-\frac{K_{q}}{2} Q_{h}(x)=3 x_{1}^{2}+3 x_{2}^{2}-x_{1}+x_{2}-3 .
$$

Its minimum on $T$ is $\left(\frac{1}{6},-\frac{1}{6}\right)$ and $\operatorname{LB}_{q}\left(\frac{1}{6},-\frac{1}{6}\right)=-3.166$.

$$
\mathrm{LB}_{\alpha}(x)=f(x)-\frac{K_{\alpha}}{2} Q_{h}(x)=4 x_{1}^{2}+3 x_{2}^{2}+x_{2}^{3}-\frac{1}{2} x_{1}-\frac{7}{2} .
$$

Its minimum on $T$ is $\left(\frac{1}{16}, 0\right)$ and $\operatorname{LB}_{\alpha}\left(\frac{1}{16}, 0\right)=-3.515$.

$$
\mathrm{LB}(x)=\frac{1}{2}\left(4 x_{1}^{2}+3 x_{2}^{2}+x_{2}^{3}-x_{1}+x_{2}-3\right) .
$$

Its minimum on $T$ is $\left(\frac{1}{8},-0.18\right)$ and $\operatorname{LB}\left(\frac{1}{8},-0.18\right)=-1.570$.

$$
\mathrm{UB}_{q}(x)=L_{h} f(x)+\frac{K_{\alpha}}{2} Q_{h}(x)=-3 x_{1}^{2}-3 x_{2}^{2}+x_{2}+4 .
$$

Its maximum on $T$ is $\left(0, \frac{1}{6}\right)$ and $\mathrm{UB}_{q}\left(0, \frac{1}{6}\right)=4.083$.

$$
\mathrm{UB}_{\alpha}(x)=f(x)+\frac{K_{q}}{2} Q_{h}(x)=-2 x_{1}^{2}-3 x_{2}^{2}+x_{2}^{3}+\frac{1}{2} x_{1}+\frac{7}{2} .
$$

Its maximum on $T$ is $\left(\frac{1}{8}, 0\right)$ and $\mathrm{UB}_{\alpha}\left(\frac{1}{8}, 0\right)=3.531$.

$$
\mathrm{UB}(x)=\frac{1}{2}\left(-2 x_{1}^{2}-3 x_{2}^{2}+x_{2}^{3}+x_{2}+4\right) .
$$

Its maximum on $T$ is $(0,0.18)$ and $\mathrm{UB}(0,0.18)=2.044$.

We obtain the following ranges of $f(x)$ : 
TABLE 1. Comparison results of the proposed bounding functions with quadratic bounding functions, the $\alpha$ bounding functions, and the best range given in [6] for computing a range of a set of test functions found in [6].

\begin{tabular}{llllll}
\hline \hline Examples & {$\left[\mathrm{LB}_{q}, \mathrm{UB}_{q}\right]$} & {$\left[\mathrm{LB}_{\alpha}, \mathrm{UB}_{\alpha}\right]$} & {$\left[\mathrm{LB}_{K}, \mathrm{UB}_{K}\right]$} & {$[\mathrm{LB}, \mathrm{UB}]$} & {$\left[\mathrm{LB}_{E}, \mathrm{UB}_{E}\right]$} \\
\hline 1 & {$[-3.166,4.083]$} & {$[-3.515,3.531]$} & {$[-4,3.5]$} & {$[-1.570,2.044]$} & {$[-0.75,1.25]$} \\
2 & {$[-1.726,2.842]$} & {$[-1.773,2.714]$} & {$[-1.75,2.625]$} & {$[-1.215,2.468]$} & {$[-0.812,1.937]$} \\
3 & {$[-0.271,0.449]$} & {$[-0.265,0.437]$} & {$[-0.282,0.411]$} & {$[-0.252,0.402]$} & {$[-0.235,0.391]$} \\
4 & {$[-23.635,25.042]$} & {$[-22.173,24.849]$} & $[-26.25 ; 24.75]]$ & {$[-12.521,16.567]$} & {$[-8.25,9.75]$} \\
5 & {$[-0.279,0.445]$} & {$[-0.268,0.431]$} & {$[-0.274,0.438]$} & {$[-0.254,0.406]$} & {$[-0.235,0.389]$} \\
6 & {$[-0.323,0.442]$} & {$[-0.229,0.465]$} & {$[-0.297,0.516]$} & {$[-0.235,0.446]$} & {$[-0.235,0.446]$} \\
7 & {$[9.138,12.281]$} & {$[9.503,12.557]$} & {$[9.22,12.49]$} & {$[9.500,12.280]$} & {$[9.50,12.28]$} \\
\hline
\end{tabular}

(1) $[-3.166,4.083]$ (with quadratic bounding functions).

(2) $[-3.515,3.531]$ (with $\alpha$ bounding functions).

(3) $[-1.570,2.044]$ (with our new bounding functions).

In the following table, we report the performance comparison results of the proposed bounding functions with quadratic bounding functions, the $\alpha$ bounding functions, and the best range given in [6] for computing a range of a set of test functions found in [6].

- $\left[\mathrm{LB}_{q}, \mathrm{UB}_{q}\right]$ is the range obtained by $\mathrm{LB}_{q}(x)$ and $\mathrm{UB}_{q}(x)$

- $\left[\mathrm{LB}_{\alpha}, \mathrm{UB}_{\alpha}\right]$ is the range obtained by $\mathrm{LB}_{\alpha}(x)$ and $\mathrm{UB}_{\alpha}(x)$

- $\left[\mathrm{LB}_{K}, \mathrm{UB}_{K}\right]$ is the best range obtained in [6] with different methods

- $[\mathrm{LB}, \mathrm{UB}]$ is the range obtained by our new bounding functions $\mathrm{LB}(x)$ and $\mathrm{UB}(x)$

- $\left[\mathrm{LB}_{E}, \mathrm{UB}_{E}\right]$ is the exact range given in $[6]$.

The results summarized in Table 1 show that our new bounding functions allow us to find a tighter range for all examples taken from [6]. For the examples 6 and 7, we find the exact ranges (i.e. $K_{\alpha}=0, \operatorname{LB}(x)=f(x)$ and $\left.\mathrm{UB}(x)=L_{h} f(x)\right)$.

\section{Conclusion}

We have proposed in this paper new lower and upper bound functions for nonconvex function over an nsimplex of $R^{n}$. We have shown that this bounding functions are tighter than the classical bounding functions. The computational results show the efficiency of these new bounding functions in computing a range of nonconvex function over simplexes. These new bounding functions can be used in a branch and bound algorithm for solving global optimization problems over simplexes, the work in this direction is currently in progress.

Acknowledgements. I would like to thank two anonymous referees for their useful comments and suggestions.

\section{REFERENCES}

[1] I.P. Androulakis, C.D. Marinas and C.A. Floudas, $\alpha$ BB: a global optimization method for general constrained nonconvex problems. J. Glob. Optim. 7 (1995) 337-363.

[2] C.A. Floudas and C.E. Gounaris, A review of recent advances in global optimization. J. Glob. Optim. 45 (3) (2009) DOI: 10.1007/s10898-008-9332-8.

[3] E. Gourdin, B. Jaumard and R. Ellaia, Global optimization of Hölder functions. J. Glob. Optim. 8 (1996) $323-348$.

[4] E.M.T. Hendrix, J.M.G. Salmeron and L.G. Casado, On function monotonicity in simplicial branch and bound. AIP Conf. Proc. 2070 (2019) 020007.

[5] M. Hladık and D. Daney, Computing the range of real eigenvalues of an interval matrix. SWIM 08, Montpellier, France, June 19-20, (2008). 
[6] S. Karhbet and R.B. Kearfott, Range bounds of functions over simplices, for branch and bound algorithms. Reliable Comput. 25, (2017) 53-73.

[7] N. Kazazakis and C.S. Adjiman, Arbitrarily tight $\alpha \mathrm{BB}$ underestimators of general non-linear functions over sub-optimal domains. J. Glob. Optim. 71 (2018) 815-844.

[8] D. Nerantzis and C.S. Adjiman, Tighter $\alpha$ BB relaxations through a refinement scheme for the scaled Gerschgorin theorem. J. Glob. Optim. 73 (2019) 467-483.

[9] M. Ouanes, M. Chebbah and A. Zidna, Combination of two underestimators for univariate global optimization. RAIRO:OR 52 (2018) 177-186.

[10] R. Paulavicius and J. Zilinskas, Simplicial Lipschitz optimization without the Lipschitz constant. J. Glob. Optim. 59 (2014) 23-40.

[11] R. Paulavicius and J. Zilinskas, Advantages of simplicial partitioning for Lipschitz optimization problems with linear constraints. Optim. Lett. 10 (2014) 1-10.

[12] R. Paulavicius, Y.D. Sergeyev, D.E. Kvasov and J. Zilinskas, Globally-biased DISIMPL algorithm for expensive global optimization. J. Glob. Optim. 59 (2014) 545-567.

[13] D.G. Sotiropoulos and T.N. Grapsa, Optimal centers in branch-and-prune algorithms for univariate global optimization. Appl. Math. Comput. 169 (2005) 247-277.

[14] X. Wang and T.S. Chang, An improved univariate global optimization algorithm with improved linear lower bounding functions. J. Glob. Optim. 8 (1996) 393-411.

[15] Y. Zhu and T. Kuno, A global optimization method, QBB, for twice-differentiable nonconvex optimization problem. J. Glob. Optim. 33 (2005) 435-464. 